\title{
Investigation of error performance in OFDM with network coding techniques in multiple relay networks
}

\author{
Ngu War Hlaing ${ }^{1}$, Ali Farzamnia ${ }^{2}$, \\ Manas Kumar Haldar ${ }^{2}$, Liau Chung Fan ${ }^{2}$
}

\begin{abstract}
The aim of this paper proposes an orthogonal frequency-division multiplexing (OFDM) with network coding to improve the error performance of the system when the messages are transmitted from user to receiver. Two-way relay (TWR) networks are applied to reduce the transmission time slots. The exclusive-OR (XOR) coding is used for network coding in which source nodes exchange their information via TWR nodes. The XOR coded bits provides redundancy to achieve the transmit diversity gain which improves the error performance of the TWR network. OFDM is exploited for TWR to obtain the frequency selective fading nature of wireless channels. The different modulation schemes such as Quadrature Phase Shift Keying (QPSK), 16-Quadrature Amplitude Modulation (QAM) and 64-QAM with OFDM system are simulated and QPSK is selected as it gives the lowest bit error rate (BER). The multiple relaying schemes with different numbers of the information packets are also considered in this paper. Simulation results show that multiple relay schemes provide faster transmission time and better error rate performance. Moreover, different kinds of channel coding schemes such as Convolutional, Reed-Solomon (RS) and turbo codes are applied in OFDM system with network coding to compare and evaluate the BER performance of the proposed system. From the simulation results, network coded OFDM scheme with turbo codes give better BER performance for given Signal-to-Noise Ratio (SNR) in relaying scheme with different numbers of information packets compared to those of convolutional and RS codes. It shows that, the error rate performance and transmission time is reduced 10 percent than the conventional scheme at even at low SNR value.
\end{abstract}

K e y w or d s: convolutional, orthogonal frequency-division multiplexing, reed-solomon and turbo codes

\section{Introduction}

In wireless communication network, the concept of cooperative communication through relays is of great interest. Network coding allows the relay to process incoming data from multiple sources so that the amount of information transmitted through the network can be reduced and the network throughput is increased $[1,2]$. In an emergency, a message consisting of voice, text and video gives a better understanding of the help needed [3,4]. The delivery time of such a large message is important. If the message can be delivered to the emergency department within the golden hours, the probability to save a life is high [5]. Hence, a low delivery time obtained by network coding is desirable. Moreover, the message should be error free as far possible. This requires channel coding.

The objectives of this research is to improve the transmission rate during data transmission through network coding and to improve bit error rate (BER) by reducing the effect of fading using orthogonal frequency division multiplexing (OFDM) and using convolutional codes, Reed-Solomon (RS) codes and turbo codes. Compared to frequency division multiplexing (FDM), OFDM also requires less bandwidth and is therefore more spectrally efficient.

\section{OFDM}

is a type of signal multiplexing that divides a high data rate modulating stream into a subsequence of symbols with each symbol of a subsequence modulating a subcarrier [6]. Mitigating the effect of fading is easier as only a few sub-carriers (SCs) are subject to fading at a given time. Every transmitter antenna would transmit the same message signal to each of the receiver antenna with different transmit paths and fading coefficients. One can combat fading by interleaving and channel coding. The orthogonal nature of the SCs can combat Inter Symbol Interference (ISI) within a subsequence. The ISI between adjacent subsequence can be eliminated by using cyclic prefix $(\mathrm{CP})$. Figure 1 explains the main concepts of an OFDM signal and the inter-relationship between the frequency and time domains. Interleaved and channel coded binary data is mapped into complex valued symbols.

After serial to parallel conversion of the symbols, the modulation of the SCs by the symbols is obtained by an inverse fast Fourier transform (IFFT). The CP is appended to the IFFT output and the result converted to a serial stream. This stream is input to a digital to analog (D/A) converter to obtain a time domain signal which can be shifted upward in frequency. At the receiver, the signal is down shifted in frequency and converted to digital by analog to digital (A/D) conversion. A serial to parallel

\footnotetext{
${ }^{1}$ Electronic System Engineering Program, Malaysia-Japan International Institute of Technology, Kuala Lumpur, Malaysia, ${ }^{2}$ Electrical and Electronic Engineering Program, Faculty of Engineering, Universiti Malaysia Sabah, Sabah, Malaysia, * Corresponding author: alifarzamnia@ieee.org
} 

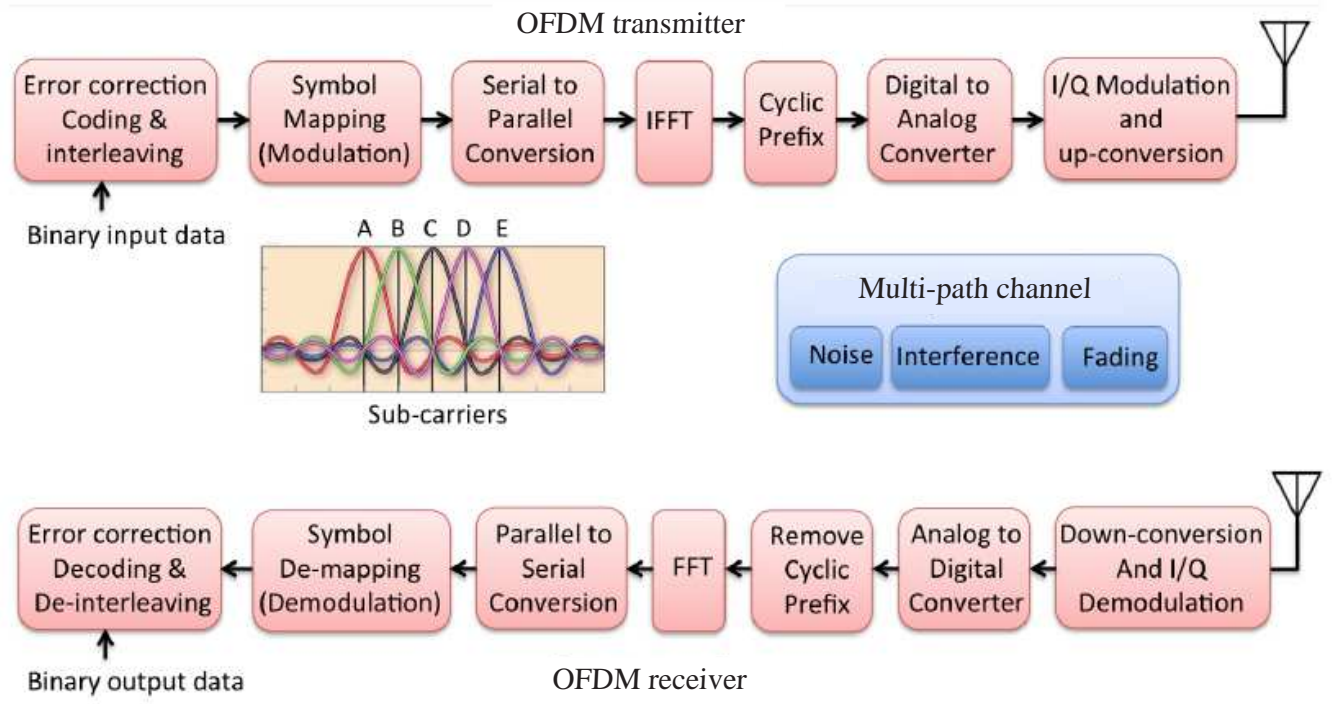

Fig. 1. The schematic of OFDM system, [5]

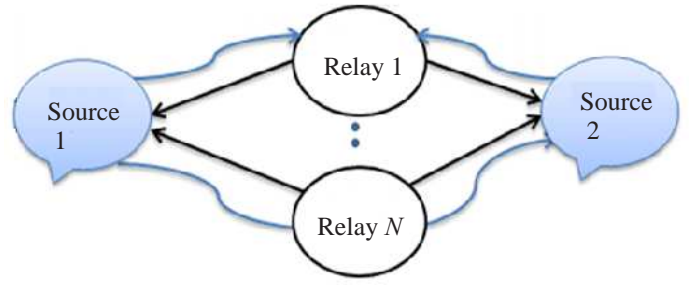

Fig. 2. Two-way relay network with 2 sources and $\mathrm{N}$ relays [6].

conversion is carried out and the cyclic prefix discarded. An operation of fast Fourier transform (FFT) then recovers the symbols in parallel form which is converted to serial and de-mapped. The output is subjected to error correction decoding and de-interleaving to get message bits. Note that the error performance also depends on the type of mapping (modulation), such as QPSK, 16-QAM etc.

\section{Network coding}

XOR network coding is the recent field of information theory. It is an archetype in which intermediate nodes are allowed to create new packets by combining (XOR) the incoming packets which provides the possibility to maximize network throughput and reduce number of transmissions [6]. The XOR coded bits provide redundancy to achieve the transmit diversity gain. The diversity gain improves the error performance of the TWR network. XOR network coding algorithm is essential mechanism to minimize packet transmissions. In [7], TWR model is used when there are two messages need to be received and sent in two time-slots in Fig. 2.

Figure 2, shows the two phases in TWR model. In phase 1, all the source nodes will transmit their information bits to the relay in first time-slot. The relay will broadcast the information bits from each source node and extract their original information signals in second timeslot in phase 2 . In this research work, convolutional codes, Reed-Solomon and turbo codes are investigated with the network coded OFDM system for encoding and decoding process.

System model of OFDM with network coding tech-
niques

After encoding, the encoded bits will be exclusive-OR coding at the source nodes. The two source nodes $A$ and $B$ exchange their information packets over a relay node $S$ [8]. So, $A$ sends the information $a$ to relay node $S$ and $B$ sends the information $b$ to relay node $S$.

Relay node, $a \otimes b$ is to recover the packets with the transmission reduction. If the relay broadcasts the $a$ or $b$, the transmission does not reach the destination in Fig. 3. But, if the base station broadcasts $a \otimes b$, the transmission will produce new information to all active nodes. This is the significant performance improvements of XOR network coding [9]. The steps of network coding scheme with OFDM with TWR network using different coding techniques are shown in Fig. 4.

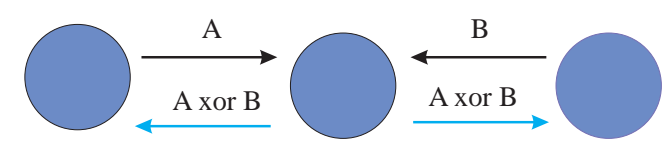

Fig. 3. Block diagram of XOR network coding, [5]

After the transmitted data source being encoded through encoding, the serial stream data input is converted into parallel data stream according to the number of the transmit antennas at the transmitter in order to increase the data rate in transmitting the data source from the transmitter to the destination [10]. Then, the parallel stream data are being modulated through modulation to enable the data source transmitting with the carrier wave through the communication channel. In the channel, the transmitted signal is attenuated through the 


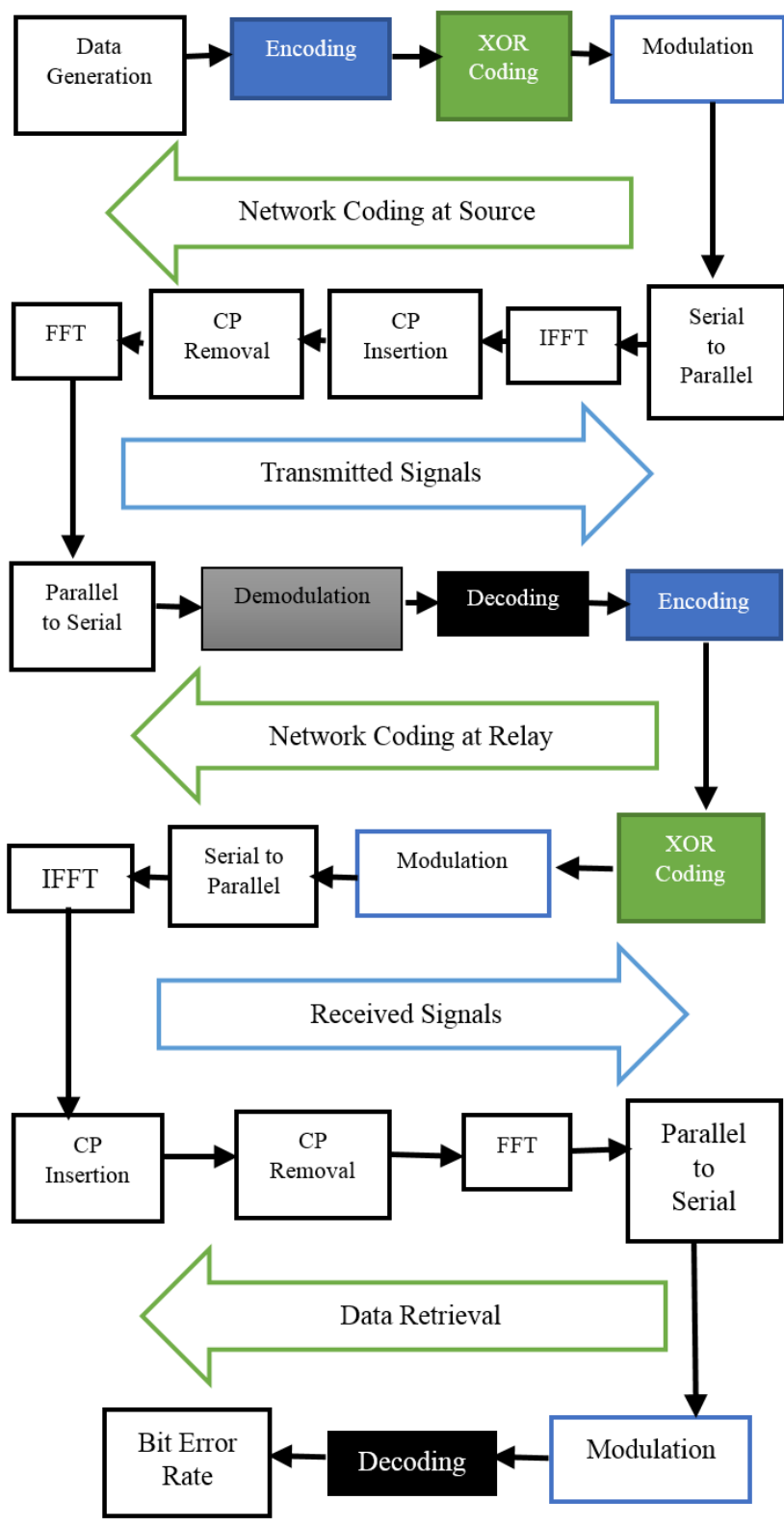

Fig. 4. Block diagram of network coding scheme with OFDM for TWR network using different coding technique, [11]

channel fading coefficient and the additive white Gaussian noise before reached the relay. The received signal at the relay from the source is described as

$$
y_{r, s}=h_{i} \times s_{t}+n_{i}
$$

where, $y_{r, s}, h_{i}, s_{t}$ and $n_{i}$ are described, $y_{r, s}$ is the received signal at the relay node, $h_{i}$ is the fading channel coefficient for different paths, $s_{t}$ is the modulated signal at the transmitter, $n_{i}$ is the additive white Gaussian noise for different paths.

\section{Combined system model of OFDM with channel and network coding technique}

We combine the OFDM system, channel and network coding into a single proposed system in TWR network. Consider an OFDM system; there are two source nodes, relay nodes and number of SCs. To create a TWR network, the packets (information bits) are exchanged by source nodes through relay nodes. The channel encoding will be applied on the information bits generated by each source node and grouped into packets, denotes as $X_{m}(1), X_{m}(2) \ldots$ which are called source nodes. Then, these source node packets are bit-wise XOR coded [11].

$$
X_{m}(1) \otimes X_{m}(2) . . \otimes X_{m}(L)=X_{m}(L+1) .
$$

Here, $L$ represents the total number of XOR coded packets. The output of XOR coded packets will apply redundancy at the transmission level. These information bits go through modulation and OFDM system. The source nodes transmit the signal to each relay node which arrives at relay nodes. The process of removing $\mathrm{CP}$ and the operation of K-point fast Fourier transform (FFT) are taken. Each source node will recover the symbols at each relay node from sub-carriers. By using XOR coded packet, information packets are processed on detection. For all coded source bits, the soft decisions are produced by the detection. Then, they are transmitted on source nodes to relay link and they come after the decoding of source bits at each relay nodes.

The network coding (XOR) is performed by each relay nodes on the detected bits from both sources [12]. The process of convolutional encoding goes through on the resulting relay bits and becomes information packets. The packets are XOR coded. The source nodes allow transmission redundancy at relay nodes. Then, modulation goes through on the coded bits resulting information symbols to be transmitted to both sources in phase 2 . These modulated symbols are allocated by each relay nodes over the same available $K$ SCs. Then, IFFT and CP insertion is operated. In phase 2 , in consecutive time slots, the correlative information is broadcasting by each relay node with OFDM over $K$ SCs.

After receiving these broadcasting signals, the removal of $\mathrm{CP}$ and K-point FFT of the received signals is processed. Each relay nodes symbols are recovered from $\mathrm{K}$ SCs and information detection is applied by using all relay signals [13]. By applying XOR coding on the decoded relay bits with its own bits, the other sources are recovered from each source [14]. Finally, the information exchange is successfully done in the TWR network.

\section{Relay detection model}

During phase 1 , on the number of SCs, the received signal at the relay node can be expressed as $X s m[k]$ is the transmitted symbol by source nodes on the $k$ number of SCs [15] where $k=1, \ldots, K, P$ is the average transmitted power of each source node. The simulation with MATLAB will be done with the QPSK modulation with gray mapping and frequency selective Rayleigh fading channel of unity power throughout the simulations. The log-likelihood ratios (LLRs) detection rule will be 


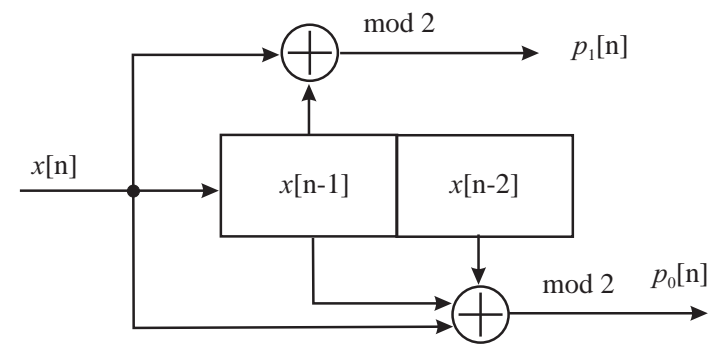

Fig. 5. Block diagram of convolutional coding with shift registers [19].

employed in this paper for the coded information bits from sources to relay, [16]. Let be

$$
\begin{gathered}
a=\left|y_{S_{m} R_{n}}\left[k_{1}\right]-\sqrt{P} h_{S_{m} R_{n}}\left[k_{1}\right] x_{S_{m}}\left[k_{1}\right]\right|^{2} \\
b=\left|y_{S_{m} R_{n}}\left[k_{2}\right]-\sqrt{P} h_{S_{m} R_{n}}\left[k_{2}\right] v_{S_{m}}\left[k_{2}\right]\right|^{2}, \\
\lambda_{S_{m} R_{n}}\left[k_{1}\right]=\min _{x_{S_{m}} \in x^{+}}\{a+b\}-\min _{x_{S_{m}} \in x^{-}}\{a+b\} .
\end{gathered}
$$

The signals received from all relay nodes process through LLRs detection and finally they are added as

$$
y_{S_{m} R_{n}}[k]=\sqrt{P} h_{S_{m} R_{n}}[k] X_{S_{m}}[k]+n_{S_{m} R_{n}}[k] .
$$

This detection will only apply when there is $R_{n}$ redundancy packet otherwise the detection is performed in the typical manner [17].

In link $R_{n} \rightarrow S_{m}$, after transmission of symbols on the detection rule to yield LLRs at $S_{m}$ can be obtained as

$$
\begin{gathered}
c=\left|y_{R_{n} S_{m}}\left[k_{1}\right]-\sqrt{P} h_{R_{n} S_{m}}\left[k_{1}\right] x_{R_{n}}\left[k_{1}\right]\right|^{2} \\
d=\left|y_{R_{n} S_{m}}\left[k_{2}\right]-\sqrt{P} h_{R_{n} S_{m}}\left[k_{2}\right] v_{R_{n}}\left[k_{2}\right]\right|^{2}, \\
\lambda_{R_{n} S_{m}}\left[k_{1}\right]=\min _{x_{R_{n}} \in x^{+}}\{c+d\}-\min _{x_{R_{n}} \in x^{-}}\{c+d\} .
\end{gathered}
$$

In phase 2 , the transmitted signal can be modelled as

$$
y_{R_{n} S_{m}}[k]=\sqrt{P} h_{R_{n} S_{m}}[k] X_{R_{n}}[k]+n_{R_{n} S_{m}}[k] .
$$

\section{Channel coding}

To get effective transmission system, various types of encoding techniques should be applied [18]. The redundancy is the key in error correction techniques. The redundancy is extra bits which is sent along with original message and it will use for error correction and detection. These bits are added by sender and removed by receiver. To obtain desired error performance, the convolutional, $\mathrm{RS}$ and turbo channel encodings are integrated in the system by assuming different numbers of relay node(s) and different number of packets.

Below, the mechanism of convolutional codes is explained for the RS codes and for the mechanism turbo codes respectively.

\subsection{Convolutional codes}

Convolutional codes, the information bits are spread along the sequence. The output of a convolutional encoder depends not only on the current input information, but also on previous inputs or outputs, either on a block-byblock or a bit-by-bit basis as shown in Fig. 5 .

A shift register is the sequence of flip-flops where the output of $n^{\text {th }}$ flip-flop is connected to the input of the $(n+1)^{t h}$ flip-flop. In convolutional encoding [19], each register has 1 bit input and starts with 0 values. The convolution of $g$ (generator polynomials) and $x$ (sequence of messages) equation is denoted as follows

$$
p_{i}[n]=\sum_{j=0}^{k-1} g_{i}[j] x[n-j] .
$$

Convolutional coding with Viterbi decoding is applied in forward error correction technique.

\subsection{Reed-Solomon codes}

The Reed-Solomon (RS) codes are burst error correcting codes because they encode the data on multi-bit symbols instead of individual bits [20]. It is one of the flexible codes because it applies one code for both error detection and correction. From the number of parity bits, we can calculate how many number of burst error can be corrected. Reed Solomon code is based on the galois field (GF) arithmetic.

Galois field consists of an element which is generated from a primitive element and denoted by $\alpha$. Galois field is generated on the concept that primitive element [20] and therefore, we can write

$$
\alpha^{8}=\alpha^{4}+\alpha^{3}+\alpha^{2}+1
$$

The 8-bit symbols $(m=8)$ and the $G F\left(2^{8}\right)$ are used. The parity symbols are added to the original messages

$$
N=K+2 t
$$

where; $N$ are Transmitted symbols, $K$ are the original messages, $2 t$ are the parity symbols.

For RS encoder and decoder, a generator polynomial $g(x)$ which contains $2 t$ factors is constructed [20]

$$
g(x)=\prod_{i=0}^{2 t-1}\left(x+\alpha^{i}\right) .
$$

To encode RS code in a systematic manner, the codeword polynomials generated by a use of the division algorithm. Dividing the shifted message polynomial $x^{d-1} m(x)$ by $g(x)$, we obtain where $g(x)$ is the generator polynomial and $p(x)$ is the parity check polynomial. The codeword vector will be

$$
c(x)=x^{d-1} m(x)+p(x),
$$




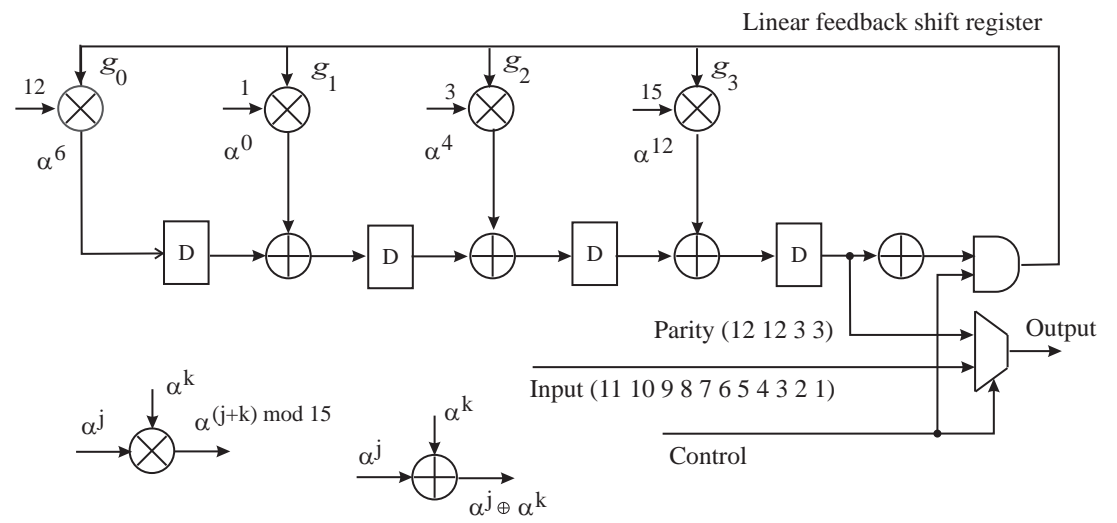

Fig. 6. Block diagram of RS encoder [20].

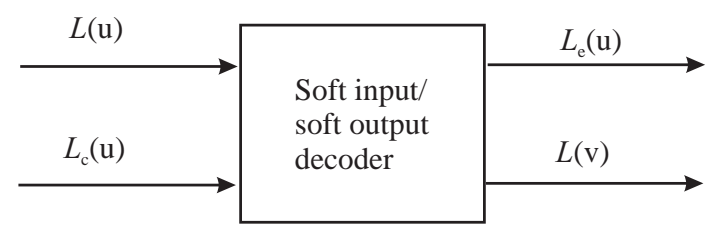

Fig. 7. Block diagram of turbo decoding [24].

$$
p(x)=x^{d-1} m(x) \operatorname{modg}(x)=\sum_{j=0}^{d-2} p_{j} x^{j} .
$$

The mechanism of RS encoder is explained in the Fig. 6 by applying the above equations.

The RS decoder can detect up to $2 t$ symbols and correct up to $t$ symbols in error at the receiver. However, when the error locations are known and such errors are called erasures, RS codes can correct up to $2 t$ erasures

$$
T(X)=R(X)+E(X),
$$

where, $R(x)$ - is the received messages, $T(x)$ - is the transmitted messages, $E(x)$ - is the error messages.

The RS decoder will identify the error messages [21]. In RS encoding, $T(x)$ is divisible by $g(x)$. If there are no errors for some (i) in $[0,, 2 t-1]$, so dividing by $g(x)$ will give the zero value. But, if there are errors for some or all terms of (i), then dividing by $\mathrm{g}(\mathrm{x})$ will give nonzero value. This value is called syndrome. The equation for syndrome is written in below. Since, a factor of $g(x)$ which is also a factor of $T(x) . S_{i}$ is

$$
S_{i}=R\left(\alpha^{i}\right)=T\left(\alpha^{i}\right)+E\left(\alpha^{i}\right)=E\left(\alpha^{i}\right) .
$$

Since, $T\left(\alpha^{i}\right)=0$ as $x+\alpha^{i}$, a factor of $g(X)$ which is also a factor of $T(X) . S_{i}$ can be written as

$$
S_{i}=Y_{1} \alpha^{i e_{1}}+Y_{2} \alpha^{i e_{2}}+\ldots+Y_{v} \alpha^{i e_{v}} .
$$

By substitutin $X_{j}=\alpha^{e_{j}}$ in equation (eq13), we can obtain

$$
S_{i}=Y_{1} X_{1}^{i}+Y_{2} X_{2}^{i}+\ldots+Y_{v} X_{v}^{i},
$$

where, $e_{1}, e_{2}, \ldots, e_{v}$ are the locations of errors and $Y_{1}, Y_{2}, \ldots, Y_{v}$ are the coefficients of errors.

\subsection{Turbo codes}

A parallel concatenation of two convolutional codes separated by a random inter-leaver is called turbo code [22]. By considering a rate $1 / 2$ recursive systematic convolutional (RSC) codes with memory size $\mathrm{M}$ and generator sequences, $g 1=(g 10, g 11, g 12, . ., g 1 v), g 2=$ $(g 20, g 21, g 22,, g 2 v), d=$ the input sequence, $S$ and $P$ represent the two output sequences [23]. A decoder could be called a soft input/hard output decoder because the final decoding process is bits

$$
\begin{aligned}
& S_{k}=\sum_{i=0}^{v} g_{1 i} d_{k-i}, \\
& Y_{k}=\sum_{i=0}^{v} g_{2 i} d_{k-i} .
\end{aligned}
$$

In Fig. $7 L(u)$ means a priori values for all information bits, $L_{c}(y)$ means channel values for all information bits, $L_{e}(u)$ means extrinsic values for all information bits and $L(v)$ means a posteriori values for all information bits. The soft output for an information bit $u$ can be expressed as, [24]

$$
L(u)=L(u \mid y)=\log \frac{P(u=+1 \mid y)}{P(u=-1 \mid y)} .
$$

Let $x=\left(x_{11}, x_{12}, \ldots, x_{1 n}, \ldots, x_{L 1} \ldots, x_{L n},\right)$ be a codeword of $L$ branches where $x_{i 1}=u_{i}$ and $x_{i 2} \ldots, x_{i n}$ are parity bits and let $\left(y_{11}, y_{12}, \ldots, y_{1 n}, \ldots, y_{L 1} \ldots, y_{L n},\right)$ be the receiver vector. Then, we can write the following equations

$$
L\left(u_{i}\right)=L\left(u_{i} \mid y\right)=\log \frac{P\left(u_{i}=+1 \mid y\right)}{P\left(u_{i}=-1 \mid y\right)}
$$

$$
\begin{gathered}
=L_{c} y_{i 1}+\log \frac{P\left(y-\left\{y_{i 1}\right\} u_{i}=+1\right)}{P\left(y-\left\{y_{i 1}\right\} u_{i}=-1\right)}+L\left(u_{i}\right), \\
=L_{c} y_{i 1}+L_{e}\left(u_{i}\right)+L\left(u_{i}\right) .
\end{gathered}
$$




\section{Results and discussion}

The performance of the proposed network coded OFDM with channel encoding techniques are evaluated and analysed through the simulation done by the MATLAB software. The performance of the simulation is tested in term of BER versus SNR (dB) and data throughput. The OFDM simulation using different modulation schemes is presented in Fig. 8.

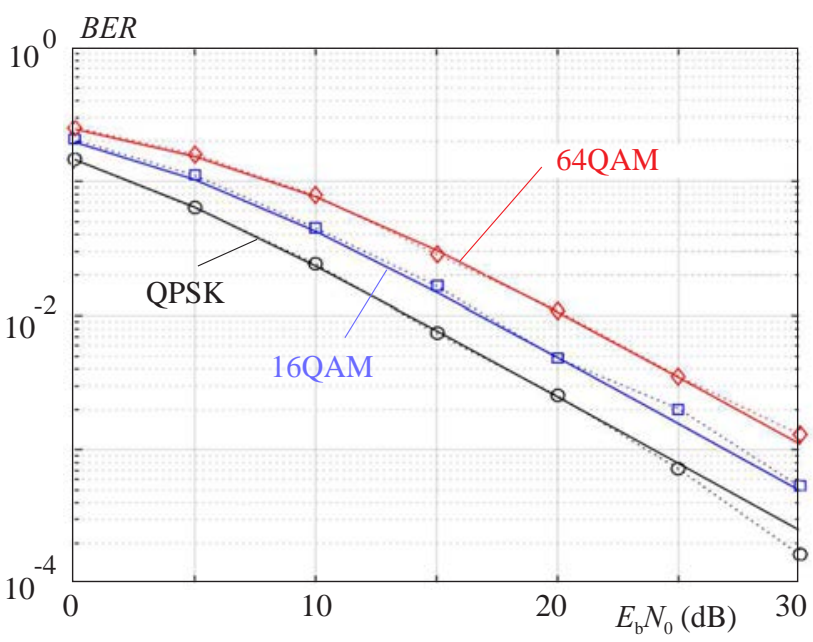

Fig. 8. Comparison of BER of different modulation techniques: simulation - $\diamond, \square, \bigcirc$, theory - solid lines

Table 1. Simulation parameters of a network coded OFDM system with different channel coding technique in TWR

\begin{tabular}{ll}
\hline Parameter & Value \\
\hline Cyclic prefixes & 16 \\
Channel & Rayleigh fading \\
Modulation order & 4 \\
Subcarriers & 256 \\
Interpolation & FFT based \\
Modulation & QPSK \\
FFT Points & 64 \\
Constraint length & 3 \\
Encoding & Convolutional, \\
Rate of coding & Half \\
Decision & Soft \\
Decoding & Viterbi, RS and turbo \\
Interleaving & Random \\
Operation mode & Continuous \\
Trace-back depth & 32 \\
\hline
\end{tabular}

Table 2. BER versus SNR (dB) of different relaying scheme using turbo coding

\begin{tabular}{lccccc}
$\begin{array}{l}\text { Redun- } \\
\text { dancy }\end{array}$ & 10 & 15 & 20 & 30 & 40 \\
\hline No & 0.4864 & 0.4036 & 0.2245 & 0.0129 & 0.0114 \\
Yes & 0.0351 & 0.0292 & 0.0234 & 0.0117 & $1.2 \times 10^{-3}$ \\
\hline
\end{tabular}

Table 3. BER versus SNR (dB) of different relaying scheme with RS code

\begin{tabular}{cccccc} 
& \multicolumn{5}{c}{$S N R(\mathrm{~dB})$} \\
Relays & 10 & 15 & 20 & 30 & 40 \\
\hline 2 & 0.0228 & 0.0183 & 0.0155 & 0.0073 & $7.55 \times 10^{-4}$ \\
3 & 0.0196 & 0.0154 & 0.0140 & 0.0062 & $6.20 \times 10^{-4}$ \\
\hline
\end{tabular}

Table 4. BER versus SNR (dB) of different relaying scheme using turbo coding

\begin{tabular}{cccccc} 
& \multicolumn{5}{c}{$S N R(\mathrm{~dB})$} \\
Relays & 10 & 15 & 20 & 30 & 40 \\
\hline 2 & 0.0193 & 0.0155 & 0.0155 & 0.0062 & $6.53 \times 10^{-4}$ \\
3 & 0.0134 & 0.0111 & 0.0088 & 0.0046 & $4.61 \times 10^{-4}$ \\
\hline
\end{tabular}

Table 5. Frame error rate performance on the diversity order

\begin{tabular}{lcccc} 
Diversity order & 3 & 5 & 7 & 9 \\
\hline FER & $10^{-12}$ & $10^{-20}$ & $10^{-28}$ & $10^{-36}$ \\
\hline
\end{tabular}

In Fig. 8, the BER performance and signal to noise ratio (Eb/No) of QPSK, 8, 16 and 64 QAM-OFDM in the Rayleigh channel are simulated. The simulation results show that the modulation order is directly proportional to the BER since it can be noticed that the QPSK modulation achieves better BER performance than other QAM schemes in OFDM system. Thus, QPSK modulation is selected to apply in the proposed network coded OFDM system with different channel coding techniques.

Analysis of XOR network coding scheme with OFDM for TWR networks with Convolution codes using QPSK modulation are investigated and presented in Fig. 9 for no redundancy scheme and Fig. 10 for redundancy scheme. The results are shown for with and without redundancy schemes employed at both sources and a relay node. Without redundancy means that there is no channel encoding applied in the proposed scheme. The needed simulation parameters are listed in the Tab. 1.

The results with and without the redundancy scheme are tabulated in Tab. 2. As it is shown, the BER performance for the proposed scheme of network coded OFDM with convolutional codes in TWR network for the redundancy scheme is better than the performance of no redundancy scheme.

The simulation results of different relays for the redundancy scheme of the proposed network coded OFDM with convolutional code are presented in Fig.11. According to the shown results as the number of relay increases, the performance of BER improves. The Reed-Solomon code RS $(255,223)$ with 8 -bit symbols are applied in the 


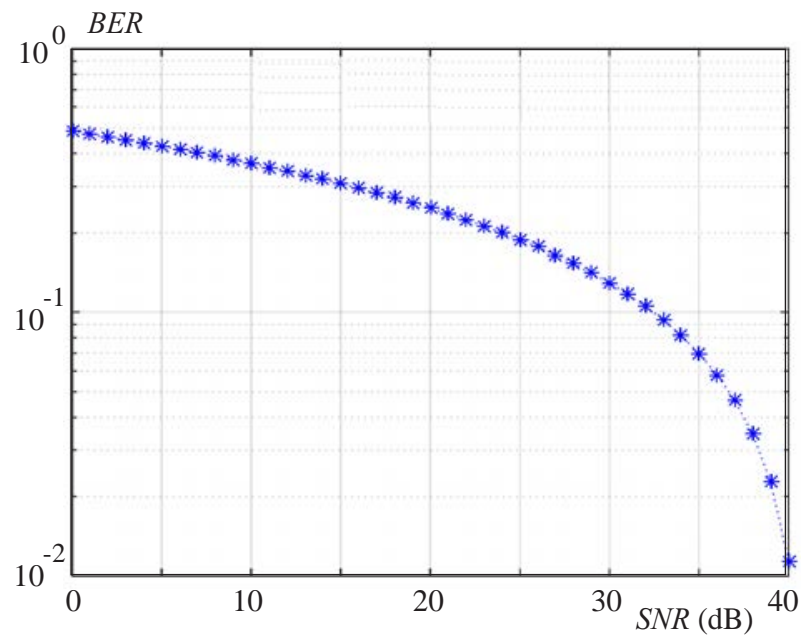

Fig. 9. Simulation result for the BER versus SNR (dB) of TWR network in OFDM systems (no redundancy)

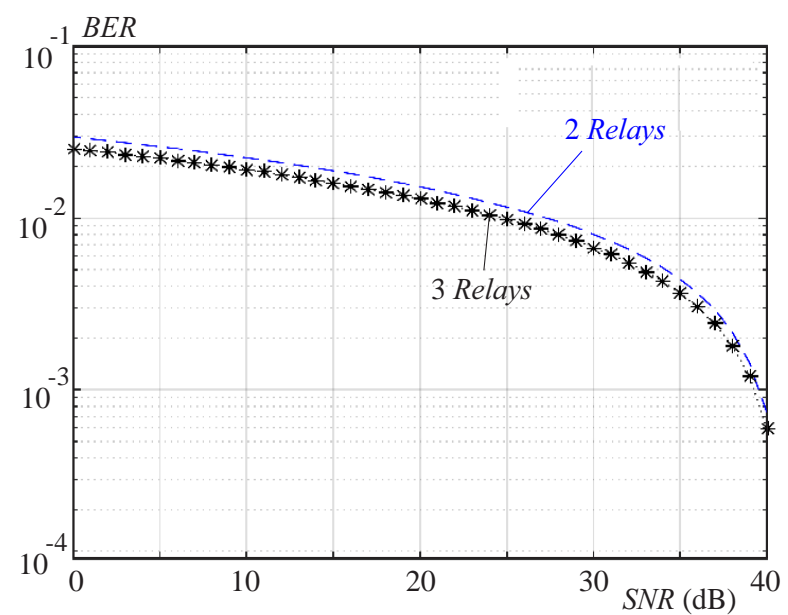

Fig. 11. Simulation result for the BER versus SNR (dB) of multiple TWR XOR network coding (redundancy at source and relay)

proposed network coded OFDM in TWR network and presented in Fig. 12.

The simulation results are shown in Fig. 12, the QPSK modulation technique shows a better error performance than other modulation techniques. Thus, QPSK modulation is chosen to apply to RS coding for multiple relay schemes in TWR network in OFDM systems. There are 255 code word bytes for each code-word, in which 223 bytes are data and 32 bytes are parity.

The simulation results for different relays for the redundancy scheme of the proposed network coded OFDM with RS code are presented in Fig. 13. The BER result for the Reed-Solomon codes with using different numbers of relays are in Tab. 3.

Although compared with the convolutional XOR coding scheme, BER for RS codes is not good as convolutional codes and the RS coding scheme does not perform considerably well as convolutional coding scheme, but the RS codes are used to correct burst errors and the coding rate is very high for Reed-Solomon code thus it is applicable in data storage and transmission.

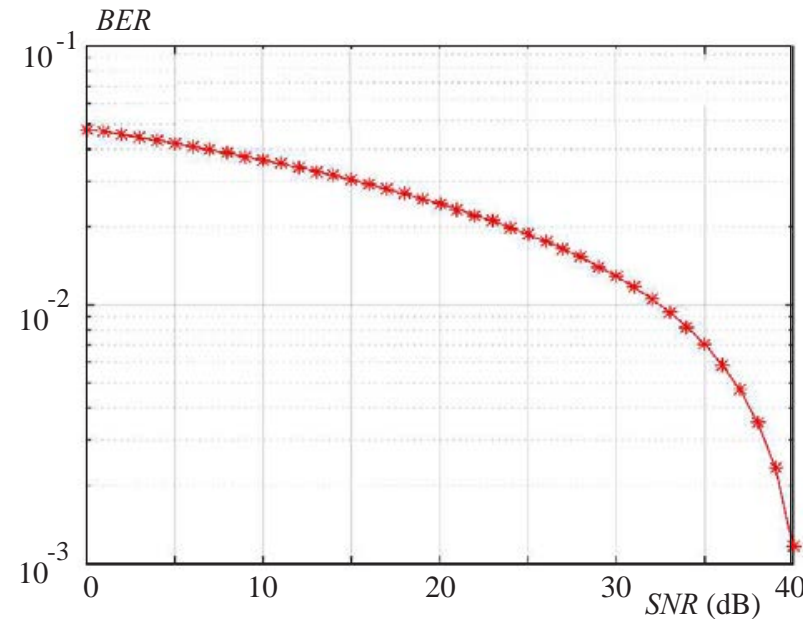

Fig. 10. Simulation result for the BER versus SNR (dB) of TWR XOR network coding (redundancy at source and relay).

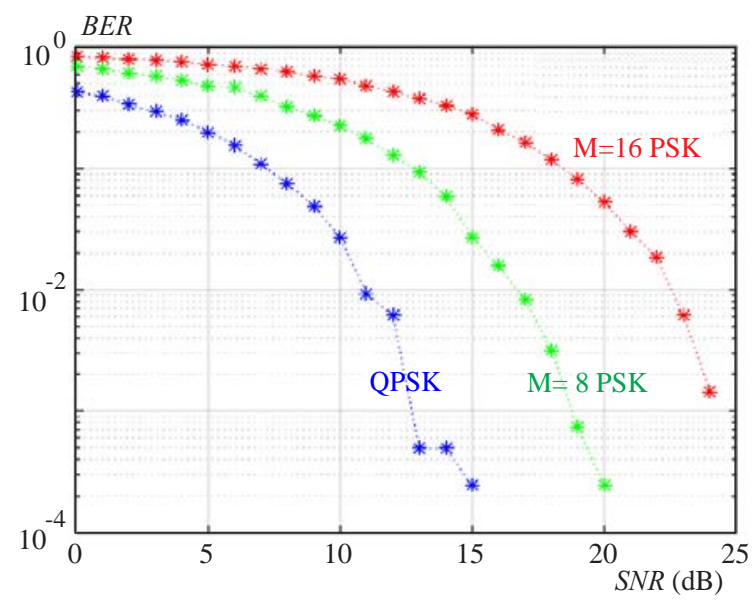

Fig. 12. Simulation result for the BER versus SNR (dB) of OFDM systems with Reed-Solomon coding

The simulation results of the conventional turbo coding and XOR coding used in source and relay in OFDM system is investigated in Fig. 14 and Fig. 15 respectively. The performance for the turbo coding scheme with relaying scheme gives better bit error rate than without relaying scheme. Moreover, different numbers of relays are developed in Fig. 16. The two and three relay numbers are used in TWR relay network with turbo codes. In Fig. 6, the three relay scheme shows the better bit error rate than the two relay scheme. The numerical results are shown in Tab. 4. Thus, to have better reliable communication system, the more number of relays can be used.

By using multiple TWR in turbo codes, the better communication system can be implemented without message delivery delays and with less noise. It can be observed that the turbo coding gives the best system performance than other coding schemes.

Moreover, the diversity order is the number of available transmission paths in data transmission. The larger the diversity order, the greater number of transmission paths. Due to the fading at the transmission path, the 


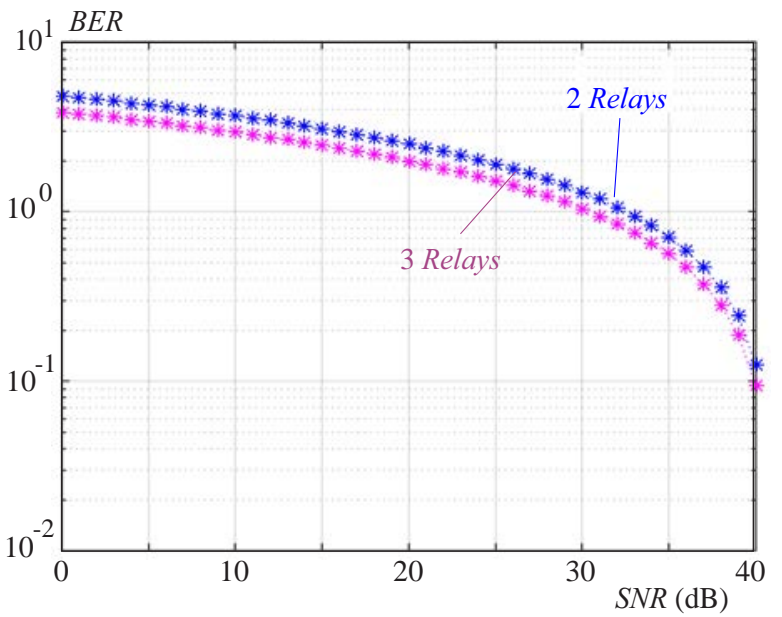

Fig. 13. Simulation Result for BER versus SNR (dB) of OFDM Systems with Reed Solomon Coding Using Different Relay Schemes.

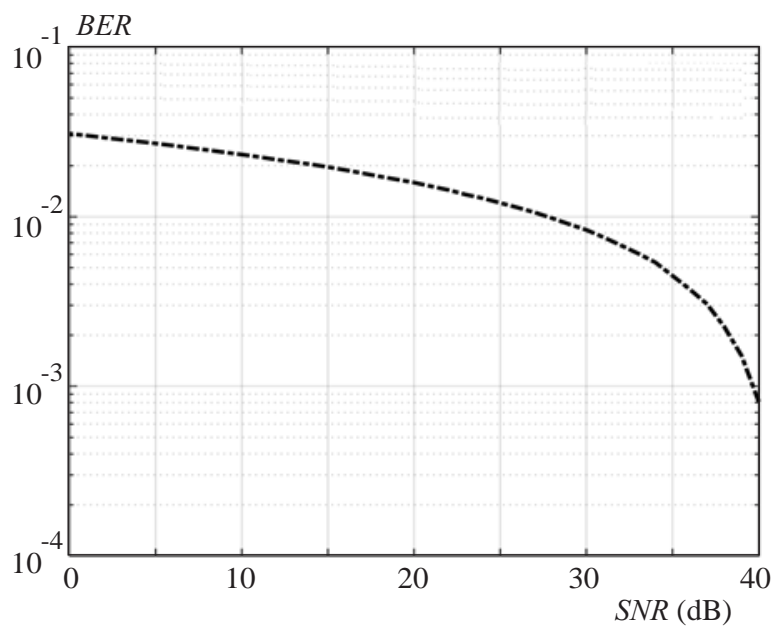

Fig. 15. Simulation result for BER versus SNR (dB) of OFDM systems with turbo coding schemes in two-way relay networks

noise interference at transmission data caused error message. Hence, diversity order in the communication system is required to against the effect that caused by fading during transmission.

The relationship between diversity order and frame error rate (FER) is tested through Fig. 17. By increasing the diversity order, the probability of error that occurred during transmission is decreased. The ratio of frames in error $F_{e}$ to the total number of frames processed at the receiver $F_{r}$ is frame error rate $F E R=F_{e} / F_{r}$.

The results show that the error probability is compensated by the diversity order, as presented in Tab. 5. In addition, diversity enables to decrease the sending signal power with same noise power in a system.

A reliable communication system can be achieved by high diversity order system. The multiple relaying schemes are implemented and simulated in Fig. 17 and it shows that the error rate becomes decreased when the number of relays is increased.

The error rate performance on the SNR $(\mathrm{dB})$ in relaying scheme with different numbers of information packets is shown in Fig. 18. The BER result for the 3 and 4 infor-

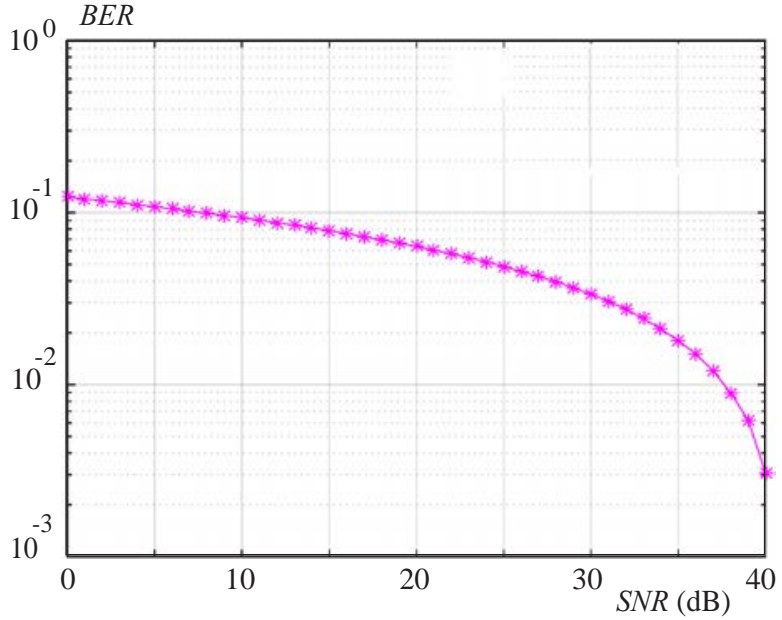

Fig. 14. Simulation Result for BER versus SNR (dB) of OFDM Systems with turbo Coding Schemes.

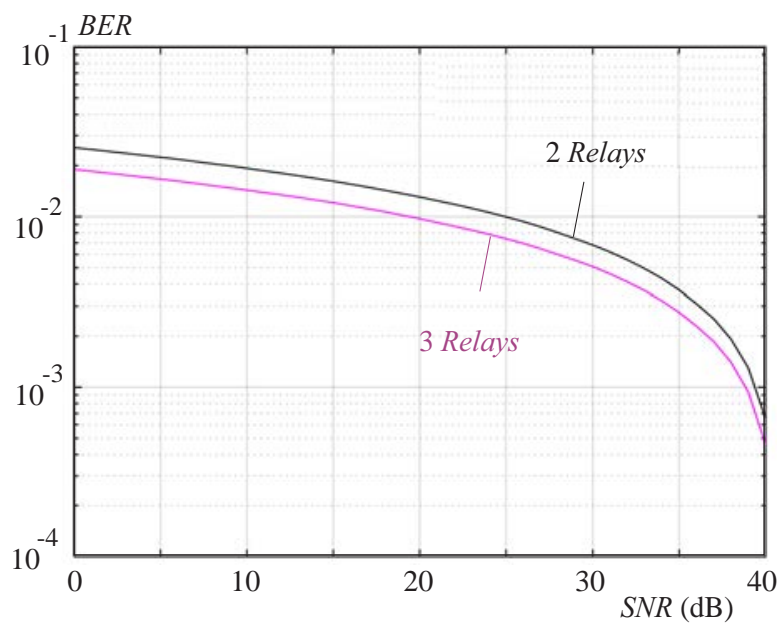

Fig. 16. Simulation result for BER versus SNR (dB) of turbo coding in two-way relay networks with multiple relays in OFDM Systems

mation packets with using different numbers shows that the performance for the larger number of relays is better compared with the smaller number of relays. And the number of increasing packets gives the bigger BER values.

\section{Conclusion}

In this paper, the simulation results are shown using OFDM system for the two-way relay network model with and redundancy schemes of network coding and channel coding techniques. Exclusive-OR (XOR) coding method combined with channel coding is applied in OFDM system with TWR network. Convolutional, RS and turbo coding are investigated to compare the system performance in two-way relay networks. The BER performance of the proposed turbo coding scheme is achieved better compared to the convolutional and RS encoding scheme. It shows that, the error rate performance and transmission time is reduced 10 percent than the conventional scheme at even at low SNR value. Moreover, it can be concluded that if more number of relays applied in the network, the number of BER became reduced. 


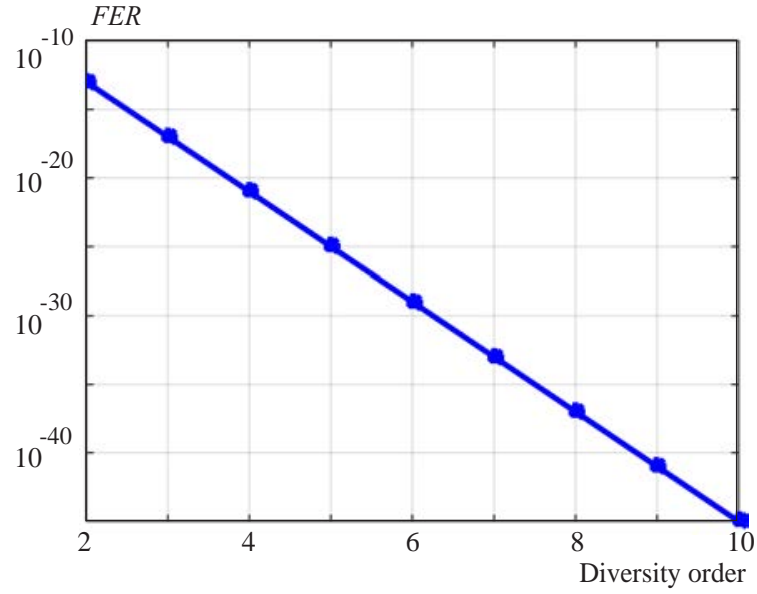

Fig. 17. Relationship between $F E R$ and diversity order

\section{Acknowledgments}

This research is supported by the Center for Research and Innovation (PPPI) and Faculty of Engineering, Universiti Malaysia Sabah (UMS).

\section{REFERENCES}

[1] W. Meng, D. Wübben, and A. Dekorsy, "Mutual information based analysis for physical-layer network coding with optimal phase control", SCC 2013; 9th International ITG Conference on Systems Communication and Coding, pp. 1-6, VDE, 2013.

[2] L. Lijun, R. Gu, Y. Ji, L. Bai, and Z. Huang, "All-optical OFDM network coding scheme for all-optical virtual private communication in PON", Optical Fiber Technology, vol. 20, no. 2, pp. 61-67, 2014.

[3] G. H. Singh and M. Singh, "Optimization Method for Analysis of Bit Error Rate with BPSK Modulation Technique", International Journal of Scientific and Engineering Research, vol. 3 no. 6, pp. 1-4, 2012.

[4] S. Saravanan, P. Harikrishna, and J. Vaideeswaran, "Big Data Exchange be-tween ambulance bus to hospital network through internet in telemedicine using computer communication network and 3G mobile antenna", International Conference on Computer Communication and Informatics, pp. 1-7, 2015.

[5] Y. Yogita, P. Mohapatra, S. Jha, and A. Seneviratne, "Received signal strength indicator and its analysis in a typical WLAN system", (short paper) 38th Annual IEEE Conference on Local Computer Networks, pp. 304-307. IEEE, 2013.

[6] A. Farzamnia, N. W. Hlaing, L. E. Kong, M. K. Haldar, and T. Y. Rezaii, "Investigation of error performance in network coded MIMO-VBLAST wireless communication systems", Journal of Electrical Engineering, vol. 70, no. 4, pp. 273-284, 2019.

[7] A. Farzamnia, N. W. Hlaing, M. Mariappan, and L. C. Fan, "Network Coding Schemes with MIMO-NOMA for Two-Way Relay Net-works", In 2018 IEEE International Conference on Artificial Intelligence in Engineering and Technology (IICAIET), pp. 1-5, IEEE, 2018.

[8] R. Khan, I, Altunbas, and G. K. Kurt, "Enhanced XOR network coding scheme with OFDM for two-way relay networks", Telecommunications Forum (TELFOR), pp. 1-4, 2016.

[9] H. Lee and I. Lee, "New approach for error compensation in coded V-BLAST OFDM systems", IEEE Transactions on Communications, vol. 55, no. 2, pp. 345-355, 2007.

[10] J. R. Walton and J. W. Ketchum, "Qualcomm Inc, Method and apparatus for processing data for transmission in a multi-channel

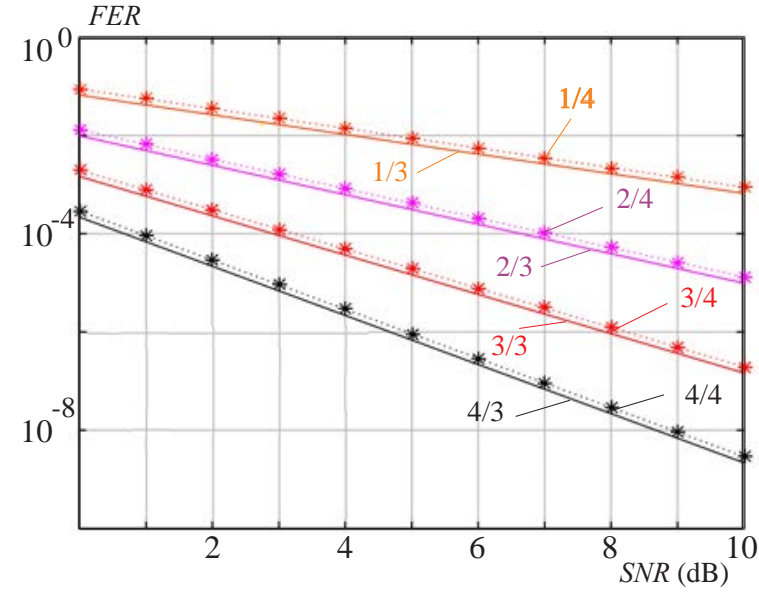

Fig. 18. Simulation result for $B E R$ versus SNR (dB) with different ratio of relays/packets

communication system us-ing selective channel transmission", US Patent 6,751,187, 2004.

[11] R. Suthar, S. Joshi, and N. Agrawal, "Performance Analysis of Different M-ARY Modulation technique in Cellular Mobile Communication", IJCA Journal IP Multimedia Communications, vol. 4 no. 1, pp. 25-29, 2011.

[12] A. Farzamnia, L. H. Zhen, and E. G. Moung, "Efficient Cellular Network with Network Coding in Disaster Area", Wireless Personal Communications, vol. 106, no. 2, pp. 701-718, 2019.

[13] I. Krikidis, J. Thompson, S. McLaughlin, and N. Goertz, "Amplify-and-forward with partial relay selection", IEEE Communications letters, vol. 12, no.4, 2008.

[14] Z. Iqbal, S. Nooshabadi, and H. N. Lee, "Analysis and design of coding and interleaving in a MIMO-OFDM communication system", IEEE Transactions on Consumer electronics, vol. 58, no. $3,2012$.

[15] L. Song, G. Hong, B. Jiao, and M. Debbah, "Joint relay selection and analog network coding using differential modulation in two-way relay channels", IEEE Transactions on Vehicular Technology, vol. 59, no. 6, pp. 2932-2939, 2010.

[16] Y. Stein, H. Malepati, and H. Primo, "Channel adaptive iterative turbo decoder system and method", US Patent 8,321,744, issued 27 November 2012.

17] X. Cai, Q. Chen, P. Fan, and Z. Ma, "On the coded complex field network coding scheme for multiuser cooperative communications with regenerative re-lays", In Communication Technology (ICCT), 2011 IEEE 13th International Conference, pp. 961-965, 2011.

18] V. Skachek, O. Milenkovic, and A. Nedic, "Hybrid Noncoherent Network Coding", IEEE Transactions on Information Theory, vol. 59, no. 6, pp. 3317-3330, 2013.

19] R. A. Baby, "Convolution coding and applications: A performance analysis under AWGN channel", In Communication Networks (ICCN), International Conference, pp. 84-88, 2015.

[20] O. O. Ogundile, Y. O. Genga, and D. J. J. Versfeld, "Symbol level iterative soft decision decoder for Reed-Solomon codes based on parity-check equations", Electronics Letters, vol. 51, no. 17, pp. 1332-1333, 2015.

[21] P. Dayal and R. K. Patial, "Implementation of Reed-Solomon CODEC for IEEE 802.16 network using VHDL code", Optimization, Reliability, and Information Technology (ICROIT), 2014 International Conference, pp. 452-455, 2014.

[22] S. Jasim and A. Abbas, "Performance of turbo code with different parameters", Journal of University of Babylon, vol. 25, no. 5, pp. 1684-1692, 2017.

[23] R. Garzón-Bohórquez, C. A. Nour, and C. Douillard, "Protograph-based interleavers for punctured turbo codes", IEEE 
Transactions on Communications vol. 66, no. 5, pp. 1833-1844, 2017.

[24] W. N. Hlaing, A. Farzamnia, M. Mariappan, and K. M. Haldar, "Network-coded MIMO-NOMA systems with FEC codes in two-way relay networks", International Journal of Communication Systems, pp. e4419.

\section{Received 19 April 2020}

Ngu War Hlaing received Bachelor of Engineering with honours and Master of Engineering in Electrical and Electronic Engineering in University Malaysia Sabah (UMS) in 2018 and 2019 respectively. Currently, she is pursuing her $\mathrm{PhD}$ in Department of Electronic Systems Engineering in MalaysiaJapan International Institute of Technology (MJIIT), University of Technology Malaysia. Her research interest includes Wireless Communications, Antenna Theory and Design, Information Theory, and Network Coding.

Ali Farzamnia received his BEng in Electrical Engineering (Telecommunication Engineering) from Islamic Azad University of Urmia IRAN 2005. He got MSc in Electrical Engineering (Telecommunication Engineering) from University of Tabriz in 2008, and obtained PhD in Electrical Engineering (Telecommunication Engineering) from Universiti Teknologi Malaysia (UTM) in 2014. He is appointed as senior lecturer (Assistant Professor) in the Electrical and Electronic Engineering program, Faculty of Engineering at Universiti Malaysia Sabah (UMS) since 2014. He is Chartered Engineer UK (CEng), senior member of IEEE and member of
IET. Dr Alis research interests are Wireless Communication, Signal Processing, Network coding, Information Theory, and Bio-Medical Signal Processing.

Manas Kumar Haldar received the $\mathrm{PhD}$ degree from the University of Cambridge, Cambridge, UK He was with the Surface Acoustic Wave Research Group, University of Oxford. He then joined the Electrical Engineering Department, National University of Singapore, Singapore. From 2006 to 2015 he was with the Swinburne University of Technology, Kuching, Malaysia, where he is now adjunct staff. From 2017, he has been with the University of Malaysia Sabah, Kota Kinabalu, Malaysia. Manas has about 38 years of teaching experience. He has published on a variety of topics, such as lightning induced over voltages, smart grid, finite elements, biometrics, optical communications, semiconductor lasers and RF filters. His current research interests are RF electronics and wireless communications.

Liau Chung Fan received the BSc degree in electrical and electronic engineering from University of Manitoba in 1992 and the MSc degrees in electrical and electronic engineering from University of Glasgow in 1998. Since 2000, he has been with Universiti Malaysia Sabah as a lecturer. His research interests include signal and image processing with a focus on its application to communication system and autonomous system perception. His industry experience includes working as an electrical maintenance engineer in a stainless steel factory and an $M \& E$ engineer in consultancy firm. 